\section{Arrhythmogenic right ventricular cardiomyopathy: From pathophysiology to diagnosis and advances in management}

\author{
Rachel Bastiaenen, Marc W. Deyell, \\ Andrew D. Krahn \\ Heart Rhythm Services, Division of \\ Cardiology, Department of Medicine, \\ University of British Columbia, \\ Vancouver, BC, Canada
}

\begin{abstract}
Our understanding of arrhythmogenic right ventricular cardiomyopathy (ARVC) has advanced considerably over the past 3040 years. This is an inherited cardiomyopathy with complicated genetic inheritance and variable penetrance. Desmosomal dysfunction underlies most cases, and appreciating this pathophysiology has contributed to patient management, particularly with respect to exercise restriction to reduce disease progression. The diagnosis is made according to a series of Task Force Criteria, and subsequent management is guided by expert consensus in the absence of comparative data. ARVC is associated with sudden cardiac death (SCD), particularly in young athletic individuals who unknowingly harbour the condition. Risk stratification is important to guide implantable cardioverter-defibrillator use and reduce SCD. Residual gaps in our understanding, particularly surrounding incomplete penetrance, the underlying pathophysiology and risk stratification, are being targeted by collaborative efforts, large registries, prospective studies and translational research.
\end{abstract}

\section{Introduction}

Arrhythmogenic right ventricular cardiomyopathy (ARVC) is an inherited cardiomyopathy associated with syncope, ventricular arrhythmias (VA) and sudden cardiac death (SCD). ${ }^{1}$ Early descriptions of the condition were based on small numbers of severely affected individuals with significant arrhythmic events and heart failure. ${ }^{2,3}$ We now have an increased understanding of the phenotypic breadth of this condition, through systematic diagnostic testing (including deep phenotyping with both imaging and electrical evaluation) coupled with family screening and genetic testing.
Desmosomal dysfunction causes disease in most patients and a pathogenic mutation can be identified in approximately $60 \%$ of those affected. ${ }^{4,5}$ Although inheritance is usually autosomal dominant, there is variable penetrance and some affected individuals may demonstrate a mild and even absent phenotype.

\section{Epidemiology}

ARVC has an estimated prevalence of 1 in 1000 to 1 in 5000; higher in European countries such as Italy and Germany. ${ }^{6}$ Patients typically present between the second and fifth decade. It is unusual for clinical signs and symptoms to occur younger than 12 or older than 60 years of age., Phenotypic expression is more prevalent in males than females, and male subjects appear more likely to develop severe disease and arrhythmic events. ${ }^{8,9}$ In some cases this sex difference relates to genotype. For example, males carrying the S358L mutation in TMEM43 develop lethal VA at a younger age than their female counterparts. ${ }^{10}$

ARVC is an important cause of SCD in the young, particularly in young athletes, where events frequently occur during exercise. ${ }^{11,12}$ Competitive sport is associated with earlier ARVC presentation and an increased risk of fatal arrhythmias. ${ }^{13}$ SCD may occur in the absence of clear phenotype and remote ARVC diagnoses have been made in some sudden unexplained death victims only by family screening and molecular autopsy. ${ }^{14}$

\section{Genetics}

Inheritance is most commonly autosomal dominant, but incomplete penetrance may obscure Mendelian patterns. ${ }^{15}$ ARVC is associated with mutations in genes encoding desmosomal proteins, most commonly plakophilin-2 [PKP2], desmoglein-2 [DSG2], desmoplakin [DSP] and desmocollin-2 [DSC2](Table 1)..$^{16-18}$ Compound and digenic heterozygosity are frequent and likely contribute to variable penetrance and the complexity of inheritance patterns. ${ }^{19}$ Increased penetrance and more severe disease have been associated with multiple mutations. ${ }^{20}$ Autosomal recessive forms are rare. These include cardiocutaneous syndromes Naxos and Carvajal disease, where cardiomyopathy is found in association with palmoplantar keratoderma and woolly hair. ${ }^{21,22}$ Causal mutations in extra-desmosomal genes have also been described.
Correspondence: Andrew Krahn, Heart Rhythm Vancouver, 211-1033 Davie Street, Vancouver, BC V6E 1M7, Canada.

Tel.: 604.682.2344, ext. 63260 - Fax: 604.806.8723.

E-mail: akrahn@mail.ubc.ca

Key words: Arrhythmogenic right ventricular cardiomyopathy; sudden cardiac death; ventricular arrhythmias.

Conflicts of interests: the authors declare no potential conflicts of interests.

Received for publication: 15 August 2017

Revision received: 10 December 2017.

Accepted for publication: 12 December 2017.

This work is licensed under a Creative Commons Attribution NonCommercial 4.0 License (CC BY-NC 4.0).

CCopyright R. Bastiaenen et al., 2017

Licensee PAGEPress, Italy

Cardiogenetics 2017; 7:6995

doi:10.4081/cardiogenetics.2017.6995

These include a Newfoundland founder population with the $\mathrm{S} 358 \mathrm{~L}$ mutation in TMEM43 which causes a fully penetrant aggressive form of the disease with high incidence of SCD and heart failure..$^{23}$

\section{Genotype-phenotype correlations}

Patients with multiple mutations have earlier occurrence of ventricular arrhythmias and higher rates of LV dysfunction and cardiac transplantation. ${ }^{24}$ Heart failure with LV dysfunction is more frequently associated with DSP than PKP2 mutations and LV involvement appears more marked in families with chain terminating mutations. ${ }^{24-26}$ Patients with PKP2 variants may have earlier onset of symptoms and VA. ${ }^{27}$ Overall, missense mutation carriers appear to have similar outcomes compared with truncating and splice site mutation carriers. ${ }^{24}$ However, there is significant variation within families. $^{28}$

\section{Pathophysiology}

Histopathology demonstrates patchy lymphocytic infiltrates, myocyte death and fibrofatty replacement of ventricular myocardium starting in the epicardial and midmyocardial layers. This leads to wall thinning and aneurysmal dilatation. The classical pattern of disease affects the right ventricle (RV) with structural changes frequently located in the subtricupsid region, 
right ventricular outflow tract (RVOT) and apex. ${ }^{29}$ This distribution is known as the triangle of dysplasia. Non-classical patterns of disease, with biventricular involvement and isolated left ventricular (LV) disease, are increasingly recognised with advances in imaging. ${ }^{30}$ Renaming the condition arrhythmogenic cardiomyopathy has consequently been suggested. ${ }^{25}$

Desmosomal dysfunction appears to be the common final pathway for most ARVC cases. Healthy desmosomes reside within the intercalated disc. These desmosomes provide mechanical continuity between myocytes and mediate intracellular and intercellular signal transduction. ${ }^{31}$ Electron microscopy studies have demonstrated remodelling of the intercalated disc and desmosomal loss in myocardial biopsies from ARVC patients. ${ }^{32}$ Immunohistochemical studies have shown that mutant desmosomal proteins shift from the intercalated disc into the cell cytoplasm and nucleus causing changes in nuclear signalling and gene transcription. ${ }^{33}$ Animal studies have suggested that genetically determined desmosomal abnormalities disrupt intercellular junctions, leading to myocyte detachment and eventual loss. Furthermore, increased expression of adipogenic and fibrogenic genes resulting from changes in nuclear signalling and gene transcription contributes to fibrofatty scarring. ${ }^{34,35}$ Mechanical stress appears to worsen desmosomal dysfunction. This may partly explain why adverse outcomes are more common in athletes with ARVC and why exercise accelerates RV dysfunction in mouse models of disease. ${ }^{36,37}$

Within the intercalated disc, the desmosome interacts with sodium channels (Nav 1.5 ) and gap junction proteins (connexin 43) to regulate adhesion, excitability and cell-to-cell coupling. This network of proteins is known as the connexome. ${ }^{31}$ Ventricular arrhythmias in ARVC appear to result from slowed cardiac conduction creating macro-reentry circuits. This may occur through areas of scar and/or as a result of gap junction remodelling leading to decreased connexin 43 and reduced sodium current. ${ }^{38,39}$ This latter theory may underlie the clinical overlap with the Brugada syndrome observed in some cases. ${ }^{40}$

TMEM43 is a nuclear membrane protein which may form part of an adipogenic pathway. ${ }^{23}$ Murine cell studies have shown that the $\mathrm{S} 358 \mathrm{~L}$ missense mutation alters the localisation of intercalated disc proteins, changes gap junction function and reduces conduction velocity. ${ }^{41}$ However, the mechanism by which this pathogenic variant causes ARVC is poorly understood at present.

\section{Clinical presentation and natural history}

Symptoms are usually due to VA and include palpitations, lightheadedness, syncope, cardiac arrest and SCD. ${ }^{5}$ SCD is an important, but uncommon, first presentation in some young adults, particularly athletes harbouring the condition. ${ }^{3}$ In diagnosed and treated ARVC patients, the long-term outcome is favourable with low rates of SCD, cardiac transplantation and cardiac mortality. ${ }^{5,24}$ Different phases are described in ARVC disease progression: the early concealed phase where individuals are usually asymptomatic but still at risk from SCD; the overt electrical phase where individuals present with arrhythmias but structurally normal hearts by conventional imaging; the structural phase where individuals exhibit ventricular morphological abnormalities; and rarely diffuse progressive disease with $\mathrm{RV}, \mathrm{LV}$ or biventricular heart failure. ${ }^{42} \mathrm{Left}$ ventricular involvement was previously thought to develop in the later stages of disease, but the advent of contrast enhanced CMR and genetic testing has shown that in some patients, particularly those with DSP mutations, LV disease occurs early and may be dominant or isolated. ${ }^{24,25,30}$ Electrical changes do appear to pre-date structural disease however. When diagnosing ARVC, abnormal imaging with a completely normal ECG should be considered suspicious and warrants careful review. ${ }^{43}$

\section{Diagnosis}

There is no single gold standard investigation for ARVC. Diagnosis is made according to a series of Task Force criteria (TFC). ${ }^{1}$ These comprise data from cardiac imaging, electrocardiography, endomyocardial biopsy (when performed), family history and genetic testing. Categories include major and minor criteria (equivalent to 2 points and 1 point respectively). Patients may be classified as definitely affected, borderline or possibly affected depending on the number of points they accumulate. A definite diagnosis requires four points (Table 2). The original 1994 TFC were based on clinical experience in patients with severe disease. ${ }^{44}$ These were highly specific but lacked sensitivity for the diagnosis of an early phenotype. In addition, qualitative analysis of CMR data resulted in ARVC over-diagnosis. ${ }^{45}$ The TFC were revised in 2010 to improve diagnostic sensitivity, albeit in the absence of a gold-standard. Revised TFC included genetic criteria, new electrical parameters and quantification of cardiac imaging. ${ }^{1}$ This led to increased ARVC diagnosis in familial disease and for carriers of desmosomal mutations, but reduced numbers of patients fulfilling CMR criteria, creating a subgroup who no longer satisfied TFC and have subsequently had their ARVC diagnosis removed. ${ }^{46}$ Unfortunately some patients underwent ICD implantation based on historic misinterpretation of CMR studies or overtreatment of benign outflow tract form of ventricular tachycardia. ${ }^{47}$

Table 1. Arrhythmogenic right ventricular cardiomyopathy (ARVC) associated genes.

\begin{tabular}{lcccc} 
Genotype & Gene & Locus & Protein & \% of disease* \\
ARVC 1 & TGFB3 & $14 \mathrm{q} 24.3$ & Transforming growth factor-beta 3 & - \\
ARVC 2 & RYR2 & $1 \mathrm{q} 43$ & Cardiac ryanodine receptor & - \\
\hline ARVC 3 & Unknown & $14 \mathrm{q} 12-\mathrm{q} 22$ & - & - \\
ARVC 4 & TTN & $2 \mathrm{q} 32.1-\mathrm{q} 32.3$ & Titin & - \\
\hline ARVC 5 & TMEM43 & $3 \mathrm{p} 25.1$ & Transmembrane protein 43 & - \\
ARVC 6 & Unknown & $10 \mathrm{p} 14-\mathrm{p} 12$ & - & - \\
\hline ARVC 7 & DES & $2 \mathrm{q} 35$ & Desmin & - \\
ARVC 8 & DSP & $6 \mathrm{p} 24$ & Desmoplakin & $2 \%-12 \%$ \\
\hline ARVC 9 & PKP2 & $12 \mathrm{p} 11$ & Plakophilin-2 & $25 \%-40 \%$ \\
ARVC 10 & DSG2 & $18 \mathrm{q} 12.1$ & Desmoglein-2 & $5 \%-10 \%$ \\
\hline ARVC 11 & DSC2 & $18 \mathrm{q} 12.1$ & Desmocollin-2 & $2 \%-7 \%$ \\
ARVC 12 & JUP & $17 \mathrm{q} 21.2$ & Plakoglobin & - \\
\hline Other & PLN & $6 q 22.1$ & Phospholamban & - \\
& LMNA & $1 \mathrm{q} 22$ & Lamin A/C & - \\
& SCN5A & $3 \mathrm{p} 21$ & Cardiac Sodium channel & - \\
& CTNNA3 & $10 \mathrm{q} 22.2$ & Alpha-T-catenin & - \\
\hline
\end{tabular}

$* \%$ of disease included where $>5 \%$; ${ }^{\circ}$ recessive form: Carvajal syndrome; ${ }^{\sharp}$ recessive form: Naxos disease. 


\section{Electrocardiographic features and ventricular arrhythmias}

Electrocardiographic (ECG) features include repolarization (T-wave inversion; TWI) and depolarization (epsilon waves, terminal activation delay of the QRS complex) abnormalities. ${ }^{48}$ TWI and epsilon waves are associated with diffuse disease and found less commonly in early, localised ARVC. ${ }^{48}$ VA include variable frequency of ventricular ectopy and ventricular tachycardia, which may degenerate into ventricular fibrillation (Figure 1). VA are often triggered by exercise and exercise testing may reveal latent disease. ${ }^{3,49}$ ECG changes are classically seen in the right precordial leads $\left(\mathrm{V}_{1}-\mathrm{V}_{3}\right)$, with extent of changes incorporated into major or minor TFC. Right ventric- ular related VA have a left bundle branch block (LBBB) morphology, with a variable axis depending on the origin or exit of the arrhythmia. In left dominant disease, ECG changes are found in the inferolateral leads and VA have right bundle branch block morphology. ${ }^{30}$ Late potentials on signalaveraged ECG are thought to represent depolarization abnormalities secondary to slowed conduction, due to myocardial scar. Although late potentials are associated with other myocardial pathologies, these have high diagnostic sensitivity and specificity when ARVC patients are compared with controls. ${ }^{50}$

\section{Cardiac imaging}

$\mathrm{CMR}$ is the preferred diagnostic imaging technique. ${ }^{43}$ It is the gold standard for cardiac volume and function assessment. Tissue characterisation, including late gadolinium enhancement (LGE) provides incremental information regarding myocardial fibrofatty scar..$^{51}$ Echocardiography allows longitudinal assessment of ventricular function, particularly for patients with implantable-cardioverter defibrillators (ICD) in situ, where CMR may be contraindicated (Figure 2). Original TFC for imaging, including myocardial fatty infiltration, were qualitative and resulted in over-diagnosis. ${ }^{44,45}$ When the TFC were revised, imaging parameters were quantified and CMR assessment of myocardial fat was no longer recommended (Table 2). ${ }^{1}$ Classical disease causes RV regional wall motion abnormalities (RWMA) with

\section{Table 2. Revised 2010 Task Force Criteria (TFC) for arrhythmogenic right ventricular cardiomyopathy (ARVC) diagnosis.}

Global or regional dysfunction and structural alterations

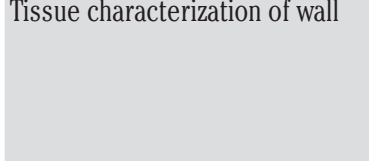

Repolarization abnormalities

abnormalities

Arrhythmias

Family history

$$
\text { Major criteria }
$$

2D echo: Regional RV akinesia, dyskinesia or aneurysm and 1 of the following (end diastole):

PLAX RVOT $\geq 32 \mathrm{~mm}\left(\geq 19 \mathrm{~mm} / \mathrm{m}^{2}\right)$

PSAX RVOT $\geq 36 \mathrm{~mm}\left(\geq 21 \mathrm{~mm} / \mathrm{m}^{2}\right)$

- Fractional area change $\leq 33 \%$

MRI: Regional RV akinesia or dyssynchronous

RV contraction and 1 of the following:

RV end-diastolic volume $\geq 110 \mathrm{ml} / \mathrm{m}^{2}$ (male)

or $\geq 100 \mathrm{ml} / \mathrm{m}^{2}$ (female)

$R V$ angiography:

Regional RV akinesia, dyskinesia or aneurysm Residual myocytes $<60 \%$ by morphometric analysis (or $<50 \%$ if estimated) with fibrous replacement of the RV free wall myocardium in $\geq 1$ sample with or without fatty replacement of tissue on endomyocardial biopsy

\section{ECG:}

- Inverted T waves in right precordial leads $\left(\mathrm{V}_{1}-\mathrm{V}_{3}\right)$ or beyond in individuals $>14$ years of age

$E C G$ : Epsilon wave (reproducible low-amplitude signals between end of QRS complex to beginning of T wave) in leads $V_{1}, V_{2}$ or $V_{3}$ (in the absence of complete RBBB with $\mathrm{QRS} \geq 120 \mathrm{~ms}$ )

Minor criteria

2D echo: Regional RV akinesia, dyskinesia or aneurysm and 1 of the following (end diastole):

- PLAX RVOT $\geq 29$ to $<32 \mathrm{~mm}\left(\geq 16\right.$ to $\left.<19 \mathrm{~mm} / \mathrm{m}^{2}\right)$

- PSAX RVOT $\geq 32$ to $<36 \mathrm{~mm}\left(\geq 18\right.$ to $\left.<21 \mathrm{~mm} / \mathrm{m}^{2}\right)$

- Fractional area change $>33 \%$ to $\leq 40 \%$

MRI: Regional RV akinesia or dyssynchronous RV contraction and 1 of the following:

- RV end-diastolic volume $\geq 100$ to $<110 \mathrm{ml} / \mathrm{m}^{2}$ (male) or $\geq 90$ to $<100 \mathrm{ml} / \mathrm{m}^{2}$ (female)

Residual myocytes $60 \%$ to $75 \%$ by morphometric analysis (or 50\% to 60\% if estimated) with fibrous replacement of the RV free wall myocardium in $\geq 1$ sample with or without fatty replacement of tissue on endomyocardial biopsy

\section{ECG:}

- Inverted $T$ waves in leads $V_{1}-V_{2}$ in individuals $>14$ years of age (in the absence of complete RBBB) or in leads $V_{4}, V_{5}, V_{6}$ - Inverted $T$ waves in leads $V_{1}-V_{4}$ in individuals $>14$ years of age in the presence of complete RBBB

SAECG: late potentials in $\geq 1$ of 3 parameters in the absence of $\mathrm{QRS}$ duration $\geq 110 \mathrm{~ms}$ on $\mathrm{ECG}$ :

- Filtered QRS duration $\geq 114 \mathrm{~ms}$

- Duration of terminal QRS $<40 \mu \mathrm{V} \geq 38 \mathrm{~ms}$

Root-mean-square voltage of terminal $40 \mathrm{~ms} \leq 20 \mu \mathrm{V}$ $E C G$ : Terminal activation duration of $\mathrm{QRS} \geq 55$ ms measured from the nadir of the $\mathrm{S}$ wave to the end of the $\mathrm{QRS}$ (including $R^{\prime}$ ) in leads $V_{1}, V_{2}$ or $V_{3}$ in the absence of complete RBBB

RVOT configuration VT of LBBB morphology with inferior axis (positive QRS in leads II, III and aVF and negative in aVL) or unknown axis

Holter: $>500$ ventricular ectopics/24 hours (negative or indeterminate QRS in leads II, II and $\mathrm{aVF}$ and positive in $\mathrm{aVL}$ )

ARVC in a first-degree relative in whom it is not possible to determine whether the family member meets current TFC Premature sudden death $(<35$ years of age) due to suspected ARVC in a first-degree relative ARVC confirmed pathologically or by current TFC in a second-degree relative relative who meets current TFC ARVC confirmed pathologically at autopsy or surgery in a first-degree relative Identification of a pathogenic mutation* categorized as associated or probably associated with ARVC in the patient under evaluation

PLAX parasternal long-axis view; RVOT, right ventricular outflow tract; PSAX, parasternal short-axis view; RBBB, right bundle branch block; VT, ventricular tachycardia. Diagnostic terminology for revised criteria: definite diagnosis: 2 major or 1 major and 2 minor criteria or 4 minor from different categories; borderline: 1 major and 1 minor or 3 minor criteria from different categories; possible: 1 major or 2 minor criteria from different categories. *A pathogenic mutation is a DNA alteration associated with ARVC that alters or is expected to alter the encoded protein, is unobserved or rare in a large non-ARVC control population, and either alters or is predicted to alter the structure or function of the protein or has demonstrated linkage to the disease phenotype in a conclusive pedigree. Adapted from Marcus et al., 2010. ${ }^{1}$ 
RV dilatation and/or impairment. CMR has higher accuracy for the detection of subtle RV abnormalities when compared with echocardiography. ${ }^{43}$ In left dominant disease, there may be LV RWMA with LV dilatation and/or impairment. LGE in the LV is usually subepicardial or midmyocardial (Figure 3). ${ }^{25,30}$ There are no diagnostic imaging criteria for left dominant disease. ${ }^{1}$ As a result, isolated $\mathrm{LV}$ disease may be attributed to myocarditis, biventricular disease may be confused with dilated cardiomyopathy (DCM), and as a result LV dominant forms are likely underdiagnosed. ${ }^{30}$

\section{Genetic testing}

In clinical practice, genetic evaluation is used for confirmatory testing in index cases and cascade screening in families. ${ }^{16} \mathrm{~A}$ pathogenic mutation can be identified in approximately $60 \%$ of index cases. ${ }^{4,5}$ Comprehensive or targeted screening of the ARVC genes can be useful for patients satisfying TFC and mutation specific screening is recommended for family members after identification of the causative mutation in an index case. ${ }^{16}$ Interpretation of ARVC genetic tests should be made by expert centres as judging the pathogenicity of a variant (particularly missense variants) can be challenging. ${ }^{16}$ Emergence of new data has led to reclassification of variants over time. ${ }^{52}$ Frequent compound and digenic heterozygosity suggests many variants alone are not sufficient for clinical disease and some rare variants have been found in healthy populations at rates high enough to downgrade the variant status from pathogenic to disease modifier or benign. ${ }^{19,52,53}$ Novel unique familial mutations are also common and these require robust determination of causality. ${ }^{54}$

\section{Differential diagnoses}

ARVC has mimics including idiopathic RVOT tachycardia, athletic heart, DCM, cardiac sarcoid, Chagas and congenital heart disease causing RV volume overload. Idiopathic RVOT tachycardia also presents with LBBB morphology VA but has a benign prognosis. The ECG morphology of ARVC-related VA have significant overlap with benign RV arrhythmias and cannot be distinguished by ECG morphology alone, although ECG features suggesting an RV free-wall origin should raise the index of suspicion of ARVC. Presence of RV structural abnormalities favours ARVC over idiopathic RVOT tachycardia, but absence of structural disease does not exclude it. Data suggest that VA with broader QRS, increasing notching and axis $<30^{\circ}$ are highly suggestive of ARVC. ${ }^{55-58} \mathrm{CMR}$ has an important role for identification of phenocopies including DCM, cardiac sarcoid and RV vol- ume overload. ${ }^{59}$ Left dominant ARVC may mimic DCM but frequent arrhythmias and subepicardial LGE on CMR favour ARVC diagnosis. ${ }^{43}$ Cardiac sarcoid may present with TWI in ECG leads $\mathrm{V}_{1}-\mathrm{V}_{3}$, ventricular arrhythmias and RV structural abnormali- ties. A combined diagnostic approach including CMR, FDG PET-CT and endomyocardial biopsy (with electroanatomic voltage mapping to improve diagnostic yield) may be required for correct diagnosis when cardiac sarcoid is suspected. ${ }^{60,61}$

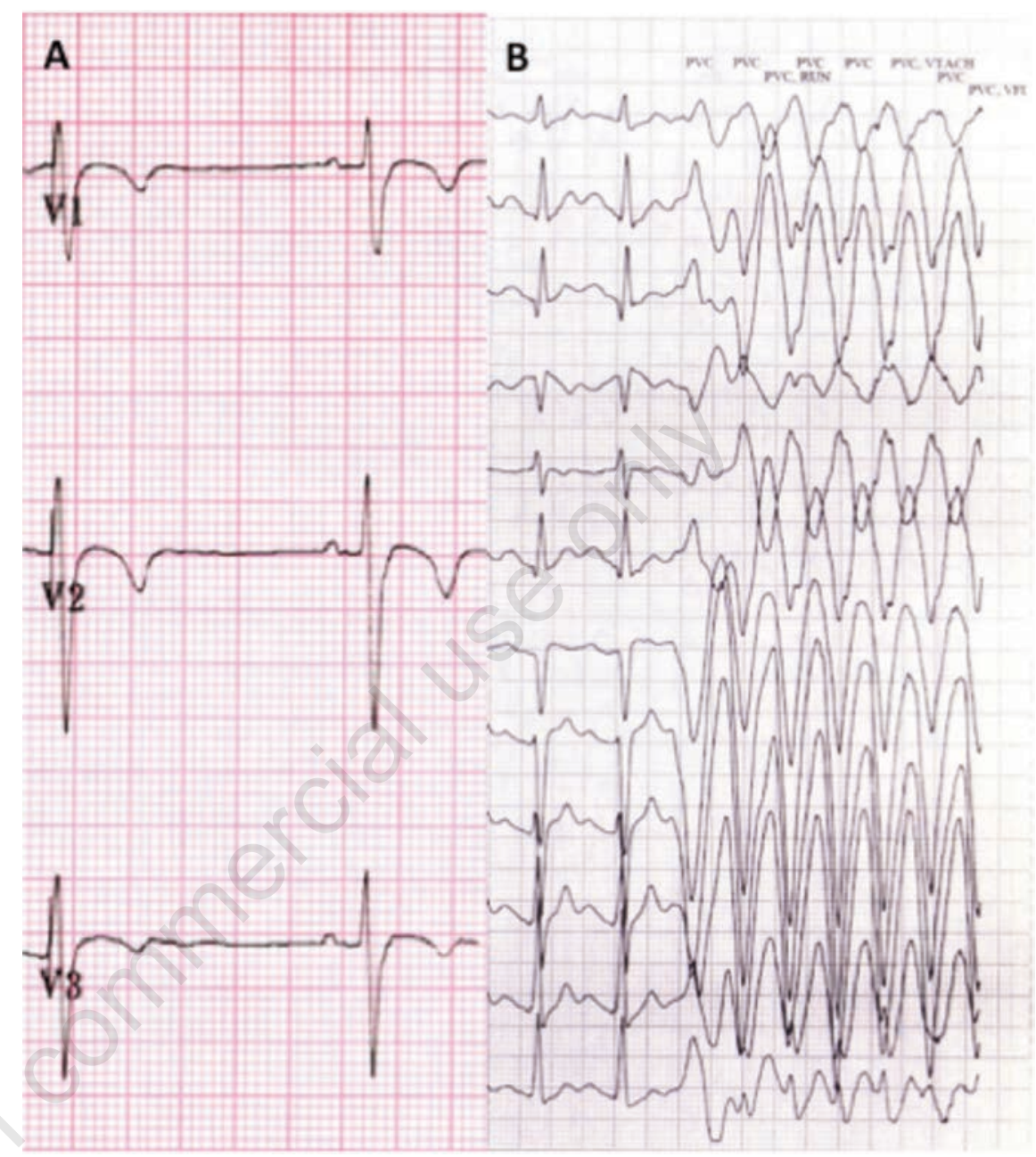

Figure 1. Electrocardiographic (ECG) features in arrhythmogenic right ventricular cardiomyopathy (ARVC). Panel (A) demonstrates $T$ wave inversion in the right precordial ECG leads $V_{1}-V_{3}$. Panel (B) demonstrates onset of left bundle branch block ventricular tachycardia with superior axis during exercise.

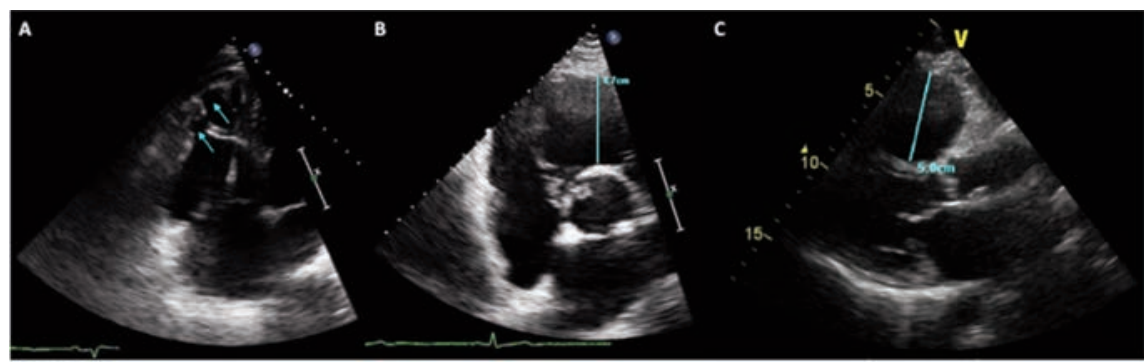

Figure 2. Echocardiography in patients with arrhythmogenic right ventricular cardiomyopathy (ARVC) demonstrates right ventricular apical aneurysms in the four-chamber view (panel $A$ ), and right ventricular dilatation in the parasternal short axis (PSAX; panel B) and parasternal long axis (PLAX; panel C) views. 


\section{Sudden death risk}

Increased risk of SCD appears to be associated with male gender, proband status, unexplained cardiac syncope, competitive and endurance exercise, extensive structural disease, electrical instability (VA and/or frequent ventricular ectopy) and multiple pathogenic variants or a mutation in TMEM43. ${ }^{13,20,23,24,62-66}$ The prognostic value of programmed ventricular stimulation in asymptomatic patients with ARVC remains unclear, but invasive evaluation of myocardial scar and fragmented, delayed electrograms may provide incremental information regarding SCD risk. ${ }^{63,64,67}$

ICD therapy reduces SCD in high-risk patients with ARVC, but ICD implantation carries risks of complication, particularly lead-related adverse events and inappropriate shocks in younger patients. ${ }^{64,68,69}$ Therefore, accurate risk stratification and appropriate patient selection is important. Newer subcutaneous ICD (S-ICD) technology represents progress towards a less invasive approach in selected patients without pacing indications. This appears safe and efficacious and may reduce complications related to transvenous leads. Not all patients are suitable however, and must be carefully screened. In addition inappropriate shock rates remain high and performance data is limited at present. ${ }^{70}$

\section{Management}

Patient management involves measures to decrease symptoms and disease progression, to protect against SCD and to organise family screening. Data regarding patient management and risk stratification in ARVC come from single centre reports and small multicentre registries. ${ }^{71}$ Patients with ARVC should be evaluated every $1-2$ years.

\section{Measures to decrease symptoms and reduce ICD therapies}

Antiarrhythmic drug therapy may improve quality of life by reducing symptomatic VA. Beta-blockers, sotalol and amiodarone are used. A combination of amiodarone with a beta-blocker appears most effective, but cumulative side effects limit long-term use of amiodarone in young patients. ${ }^{72}$ In patients with sustained monomorphic VT, invasive electrophysiological voltage mapping can identify areas of scar and guide catheter ablation of VA. This is more successful when epicardial and endocardial approaches are combined (Figure 4). ${ }^{73}$ Due to the progressive nature of disease, there may be recurrences but recent data suggests good outcomes, particularly reduction in long-term amiodarone

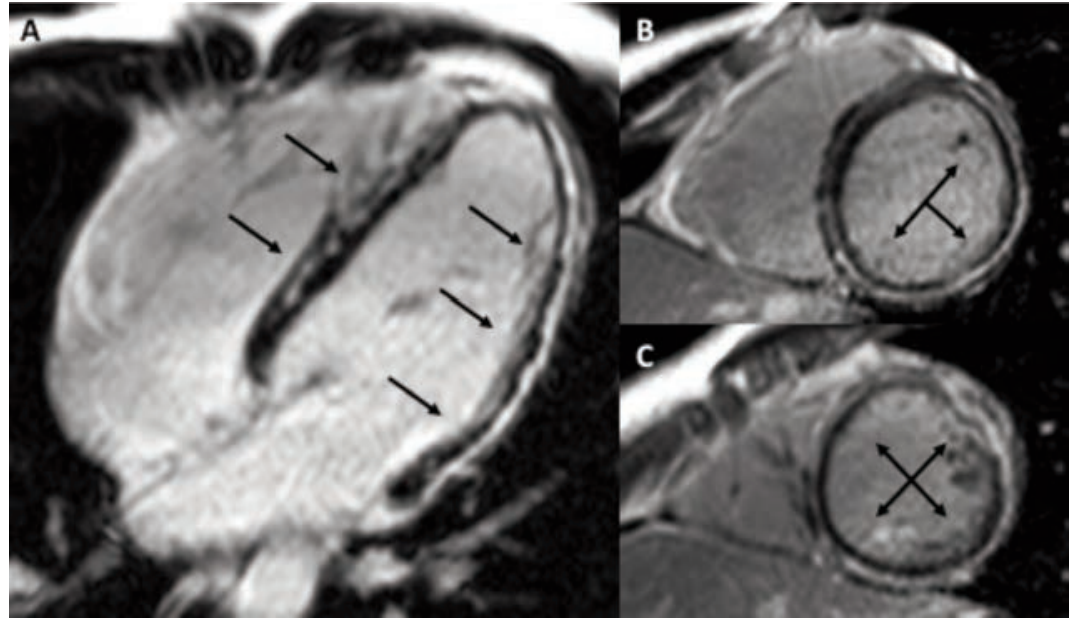

Figure 3. Cardiac magnetic resonance (CMR) imaging demonstrates subepicardial and midmyocardial left ventricular late gadolinium enhancement in a patient with arrhythmogenic right ventricular cardiomyopathy (ARVC). Panel (A) is a long axis four chamber image and panels $(B)$ and $(C)$ are short axis views.

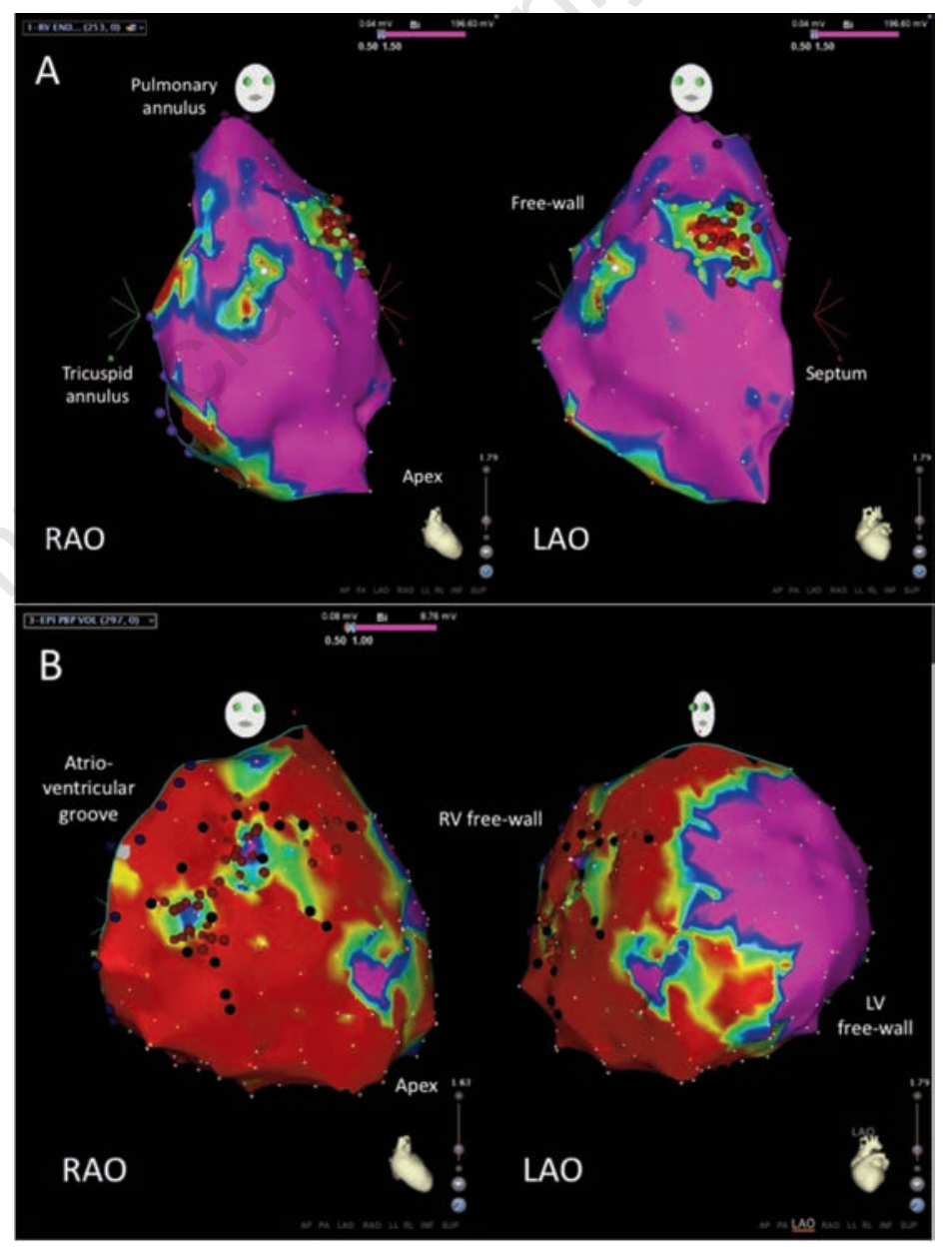

Figure 4. Endocardial and epicardial voltage mapping. Panel (A) shows right ventricular endocardial bipolar voltage maps in the right anterior oblique (RAO) and left anterior oblique (LAO) views from a 22-year-old patient with arrhythmogenic right ventricular cardiomyopathy (ARVC) and ventricular tachycardia refractory to amiodarone. Panel (B) shows epicardial bipolar voltage maps from the same views. Purple reflects healthy myocardium and red reflects severely scarred myocardium or epicardial fat. Note the patchy endocardial scar but more extensive epicardial scar. The black or green markers indicate late potentials (delayed electrical activity within the scar). The red markers are ablation lesions performed during the procedure. 
Table 3. Risk stratification and indications for implantable-cardioverter defibrillator (ICD) therapy.

\begin{tabular}{lll} 
Risk of serious arrhythmia & Markers of risk & ICD guidance \\
High risk (>10\% per year) & - Aborted SCD & ICD indicated (class I) \\
& - Sustained VT & \\
& - Severe RV and/or LV dysfunction (RV FAC $\leq 17 \%$, RVEF and/or LVEF $\leq 35 \%)$ & ICD should be considered (class IIa) \\
Intermediate risk & $\geq 1$ major risk factor & \\
& - Syncope & - Non-sustained VT \\
& - Moderate RV and/or LV dysfunction (RV FAC 17\%-24\%, & ICD may be considered (class IIb) \\
& RVEF 36\%-40\% and/or LVEF 36\%-45\%) & ICD not indicated (class III) \\
\hline Intermediate risk & $\geq 1$ other (minor) risk factor & \\
\hline Low risk (<1\% per year) & No risk factors & Healthy gene carriers
\end{tabular}

SCD, sudden cardiac death; VT, ventricular tachycardia; RV, right ventricle; LV, left ventricle; FAC, fractional area change; RVEF, RV ejection fraction; LVEF, LV ejection fraction. Adapted from Corrado et al. 2015. ${ }^{11}$

therapy. ${ }^{74,75}$ Heart failure (RV or LV) is treated with standard pharmacologic therapy including angiotensin-convertingenzyme inhibitors, angiotensin-II receptor blockers, beta-blockers and diuretics. ${ }^{71}$ Cardiac transplantation may be considered in severe disease. ${ }^{76}$ Anticoagulation is indicated for patients who develop intracardiac thrombus and/or systemic thromboembolism. ${ }^{71}$

\section{Measures to decrease disease pro- gression}

Endurance training at a competitive level has been shown to exacerbate the ARVC phenotype and therefore avoidance of high-level endurance training and competitive sports is recommended. Engagement in recreational sports with low cardiovascular demand appears acceptable. ${ }^{77}$

\section{Reduction of SCD risk}

Patients with aborted SCD and sustained VT with haemodynamic compromise are at highest risk of SCD (up to $10 \%$ per annum) and ICD insertion is recommended. ${ }^{78}$ Consensus guidelines based on extrapolation from other cardiomyopathies and personal experience also include severe ventricular dysfunction as a high-risk feature (RVEF and/or LVEF $\leq 35 \%) .{ }^{71}$ ICD therapy may be considered in patients with major risk factors including unexplained syncope, non-sustained VT and moderate RV and/or LV dysfunction. It is generally agreed that asymptomatic patients without risk factors and healthy gene carriers are at low risk of arrhythmic events and do not require ICD insertion (Table 3). ${ }^{71,78}$ In borderline patients, an implantable cardiac monitor may be considered to assess for VA and aid decisions about subsequent ICD placement.

\section{Family screening and identification of subtle disease}

Family screening comprises clinical and genetic evaluation. Clinical testing comprises ECG, echocardiography and/or CMR, Holter monitoring and exercise testing, with interpretation of results guided by the modified TFC (for familial disease). ${ }^{1,71}$ Definitive genetic diagnosis in an index case enables cascade screening of family members. Those who test positive continue clinical follow-up whereas those who test negative may be discharged. However, given the complexities of ARVC genetics and the uncertainty surrounding pathogenicity of some variants, caution should be exercised prior to discharge of gene-negative relatives. ${ }^{16}$ Family members and healthy gene carriers should be evaluated every 2-3 years. ${ }^{71}$

\section{Future directions}

In a rare disease without randomised controlled trial data, the evidence base for guidelines is limited. There are unanswered questions, particularly surrounding variable penetrance, the underlying pathophysiology and risk stratification. Collaborative registries will help to address this. ${ }^{79}$ Prospective and multicentre randomised controlled studies with larger patient cohorts and longer follow-up are also needed. Therapeutic advances will depend on improved understanding of the underlying genetic and molecular mechanisms and translational work bringing together basic scientists and clinicians will be important to further advancement in this area.

\section{References}

1. Marcus FI, McKenna WJ, Sherrill D, et al. Diagnosis of arrhythmogenic right ventricular cardiomyopathy/dysplasia. Eur Heart J 2010;31:806-14.

2. Marcus FI, Fontaine GH, Guiraudon G, et al. Right ventricular dysplasia: a report of 24 adult cases. Circulation 1982;65:384-98

3. Thiene G, Nava A, Corrado D, et al. Right ventricular cardiomyopathy and sudden death in young people. N Engl J Med 1988;319:174-6.

4. Lazzarini E, Jongbloed JDH, Pilichou $\mathrm{K}$, et al. The ARVD/C Genetic Variants Database: 2014 Update. Hum Mutat 2015;36:403-10.

5. Groeneweg JA, Bhonsale A, James CA, et al. Clinical presentation, long-term follow-up, and outcomes of 1001 arrhythmogenic right ventricular dysplasia/cardiomyopathy patients and family members. Circ Cardiovasc Genet 2015;8:437-46

6. Peters S, Trümmel M, Meyners W. Prevalence of right ventricular dysplasia-cardiomyopathy in a non-referral hospital. Int J Cardiol 2004;97:499-501.

7. Dalal D, Nasir K, Bomma C, et al. Arrhythmogenic right ventricular dysplasia: a united states experience. Circulation 2005;112:3823-32.

8. Bauce B, Frigo G, Marcus FI, et al. Comparison of clinical features of arrhythmogenic right ventricular cardiomyopathy in men versus women. Am J Cardiol 2008;102:1252-7.

9. Bhonsale A, James CA, Tichnell C, et al. Risk stratification in arrhythmogenic right ventricular dysplasia/cardiomyopathy-associated desmosomal mutation carriers. Circ Arrhythm Electrophysiol 2013;6:569-78. 
10. Hodgkinson KA, Connors SP, Merner $\mathrm{N}$, et al. The natural history of a genetic subtype of arrhythmogenic right ventricular cardiomyopathy caused by a p.S358L mutation in TMEM43. Clin Genet 2013;83:321-31.

11. Corrado D, Thiene G, Nava A, et al. Sudden death in young competitive athletes: clinicopathologic correlations in 22 cases. Am J Med 1990;89:588-96.

12. Finocchiaro G, Papadakis M, Robertus JL, et al. Etiology of sudden death in sports: insights from a United Kingdom Regional Registry. J Am Coll Cardiol 2016;67:2108-15.

13. Ruwald AC, Marcus F, Estes NA $3^{\text {rd }}$, et al. Association of competitive and recreational sport participation with cardiac events in patients with arrhythmogenic right ventricular cardiomyopathy: results from the North American multidisciplinary study of arrhythmogenic right ventricular cardiomyopath. Eur Heart J 2015;36:1735-43.

14. Bagnall RD, Weintraub RG, Ingles J, et al. A prospective study of sudden cardiac death among children and young adults. N Engl J Med 2016;374:244152.

15. Sen-Chowdhry S, Syrris P, McKenna WJ. Genetics of right ventricular cardiomyopathy. J Cardiovasc Electrophysiol 2005;16:927-35.

16. Ackerman MJ, Priori SG, Willems S, et al. HRS/EHRA Expert Consensus Statement on the State of Genetic Testing for the Channelopathies and Cardiomyopathies: this document was developed as a partnership between the Heart Rhythm Society (HRS) and the European Heart Rhythm Association (EHRA). Heart Rhythm 2011;8:130839.

17. Ohno S. The genetic background of arrhythmogenic right ventricular cardiomyopathy. J Arrhythmia 2016; 32:398-403.

18. Rampazzo A, Nava A, Malacrida S, et al. Mutation in human desmoplakin domain binding to plakoglobin causes a dominant form of arrhythmogenic right ventricular cardiomyopathy. Am J Hum Genet 2002;71:1200-6.

19. Xu T, Yang Z, Vatta M, et al. Compound and digenic heterozygosity contributes to arrhythmogenic right ventricular cardiomyopathy. J Am Coll Cardiol 2010;55:587-97.

20. Rigato I, Bauce B, Rampazzo A, et al. Compound and digenic heterozygosity predicts lifetime arrhythmic outcome and sudden cardiac death in desmosomal gene-related arrhythmogenic right ventricular cardiomyopathy. Circ
Cardiovasc Genet 2013;6:533-42.

21. McKoy G, Protonotarios N, Crosby, A et al. Identification of a deletion in plakoglobin in arrhythmogenic right ventricular cardiomyopathy with palmoplantar keratoderma and woolly hair (Naxos disease). Lancet 2000;355:2119-24.

22. Norgett EE, Hatsell SJ, Carvajal-Huerta $\mathrm{L}$, et al. Recessive mutation in desmoplakin disrupts desmoplakin-intermediate filament interactions and causes dilated cardiomyopathy, woolly hair and keratoderma. Hum Mol Genet 2000;9:2761-6.

23. Merner ND, Hodgkinson KA, Haywood AFM, et al. Arrhythmogenic right ventricular cardiomyopathy type 5 is a fully penetrant, lethal arrhythmic disorder caused by a missense mutation in the TMEM43 gene. Am J Hum Genet 2008;82:809-21.

24. Bhonsale A, Groeneweg JA, James CA, et al. Impact of genotype on clinical course in arrhythmogenic right ventricular dysplasia/cardiomyopathy-associated mutation carriers. Eur Heart J 2015;36:847.

25. Sen-Chowdhry S, Syrris P, Ward D, et al. Clinical and genetic characterization of families with arrhythmogenic right ventricular dysplasia/cardiomyopathy provides novel insights into patterns of disease expression. Circulation 2007;115:1710-20.

26. Castelletti S, Vischer AS, Syrris P, et al. Desmoplakin missense and non-missense mutations in arrhythmogenic right ventricular cardiomyopathy: genotypephenotype correlation. Int $\mathrm{J}$ Cardiol 2017;249:268-73.

27. Dalal D, Molin LH, Piccini J, et al. Clinical features of arrhythmogenic right ventricular dysplasia/cardiomyopathy associated with mutations in plakophilin-2. Circulation 2006; 113:1641-9.

28. Sen-Chowdhry S, Syrris P, Pantazis A, et al. Mutational heterogeneity, modifier genes, and environmental influences contribute to phenotypic diversity of arrhythmogenic cardiomyopathy. Circ Cardiovasc Genet 2010;3:323-30.

29. Basso C, Thiene G, Corrado D, et al. Arrhythmogenic right ventricular cardiomyopathy. Dysplasia, dystrophy, or myocarditis? Circulation 1996;94:98391.

30. Sen-Chowdhry S, Syrris P, Prasad SK, et al. Left-dominant arrhythmogenic cardiomyopathy: an under-recognized clinical entity. J Am Coll Cardiol 2008;52:2175-87.

31. Delmar M, McKenna WJ. The cardiac desmosome and arrhythmogenic cardiomyopathies. Circ Res 2010;107:70014.

32. Basso C, Czarnowska E, Della Barbera $\mathrm{M}$, et al. Ultrastructural evidence of intercalated disc remodelling in arrhythmogenic right ventricular cardiomyopathy: an electron microscopy investigation on endomyocardial biopsies. Eur Heart J 2006;27:1847-54.

33. Asimaki A, Tandri H, Huang H, et al. A new diagnostic test for arrhythmogenic right ventricular cardiomyopathy. N Engl J Med 2009;360:1075-84.

34. Garcia-Gras E, Lombardi R, Giocondo $\mathrm{MJ}$, et al. Suppression of canonical Wnt/beta-catenin signaling by nuclear plakoglobin recapitulates phenotype of arrhythmogenic right ventricular cardiomyopathy. J Clin Invest 2006; 116:2012-21.

35. Lombardi R, Dong J, Rodriguez G, et al. Genetic fate mapping identifies second heart field progenitor cells as a source of adipocytes in arrhythmogenic right ventricular cardiomyopathy. Circ Res 2009;104:1076-84.

36. James CA, Bhonsale A, Tichnell C, et al. Exercise increases age-related penetrance and arrhythmic risk in arrhythmogenic right ventricular dysplasia/cardiomyopathy-associated desmosomal mutation carriers. J Am Coll Cardiol 2013;62:1290-7.

37. Kirchhof P, Fabritz L, Zwiener M, et al. Age- and training-dependent development of arrhythmogenic right ventricular cardiomyopathy in heterozygous plakoglobin-deficient mice. Circulation 2006;114:1799-806.

38. Ellison KE, Friedman PL, Ganz LI, Stevenson WG. Entrainment mapping and radiofrequency catheter ablation of ventricular tachycardia in right ventricular dysplasia. J Am Coll Cardiol 1998;32:724-8.

39. Sato PY, Musa H, Coombs W, et al. Loss of plakophilin-2 expression leads to decreased sodium current and slower conduction velocity in cultured cardiac myocytes. Circ Res 2009;105:523-6.

40. Corrado D, Zorzi A, Cerrone M, et al. Relationship between arrhythmogenic right ventricular cardiomyopathy and brugada syndrome: new insights from molecular biology and clinical implications. Circ Arrhythm Electrophysiol 2016;9:e003631.

41. Siragam V, Cui X, Masse $S$ et al. TMEM43 mutation p.S358L alters intercalated disc protein expression and reduces conduction velocity in arrhythmogenic right ventricular cardiomyopathy. PLoS One 2014;9:e109128. 
42. Corrado D, Fontaine G, Marcus FI, et al. Arrhythmogenic right ventricular dysplasia/cardiomyopathy. Circulation 2000;101:e101-6.

43. Haugaa KH, Basso C, Badano LP, et al. Comprehensive multi-modality imaging approach in arrhythmogenic cardiomyopathy-an expert consensus document of the European Association of Cardiovascular Imaging. Eur Hear J Cardiovasc Imaging 2017;18:237-53.

44. McKenna WJ, Thiene G, Nava A, et al Diagnosis of arrhythmogenic right ventricular dysplasia/cardiomyopathy. Task Force of the Working Group Myocardial and Pericardial Disease of the European Society of Cardiology and of the Scientific Council on Cardiomyopathies of the International Society. Br Heart J 1994;71:215-8.

45. Bomma C, Rutberg J, Tandri H, et al. Misdiagnosis of arrhythmogenic right ventricular dysplasia/cardiomyopathy. J Cardiovasc Electrophysiol 2004;15: 300-6.

46. Femia G, Sy RW, Puranik R. Systematic review: Impact of the new task force criteria in the diagnosis of arrhythmogenic right ventricular cardiomyopathy. Int J Cardiol 2017;241:311-7.

47. Marcus F, Basso C, Gear K, Sorrell VL. Pitfalls in the diagnosis of arrhythmogenic right ventricular cardiomyopathy/dysplasia. Am J Cardiol 2010;105: 1036-9.

48. Nasir K, Bomma C, Tandri H, et al. Electrocardiographic features of arrhythmogenic right ventricular dysplasia/cardiomyopathy according to disease severity: a need to broaden diagnostic criteria. Circulation 2004; 110:1527-34.

49. Perrin MJ, Angaran P, Laksman Z, et al. Exercise testing in asymptomatic gene carriers exposes a latent electrical substrate of arrhythmogenic right ventricular cardiomyopathy. J Am Coll Cardiol 2013;62:1772-9.

50. Kamath GS, Zareba W, Delaney J, et al. Value of the signal-averaged electrocardiogram in arrhythmogenic right ventricular cardiomyopathy/dysplasia. Heart Rhythm 2011;8:256-62.

51. te Riele AS, Tandri H, Bluemke DA. Arrhythmogenic right ventricular cardiomyopathy (ARVC): cardiovascular magnetic resonance update. J Cardiovasc Magn Reson 2014;16:50.

52. Kapplinger JD, Landstrom AP, Salisbury BA, et al. Distinguishing arrhythmogenic right ventricular cardiomyopathy/dysplasia-associated mutations from background genetic noise. J Am Coll Cardiol 2011;57:2317-
27.

53. Andreasen C, Nielsen JB, Refsgaard L, et al. New population-based exome data are questioning the pathogenicity of previously cardiomyopathy-associated genetic variants. Eur J Hum Genet 2013;21:918-28.

54. Sen-Chowdhry S, Syrris P, McKenna WJ. Role of genetic analysis in the management of patients with arrhythmogenic right ventricular dysplasia/cardiomyopathy. J Am Coll Cardiol 2007; 50:1813-21.

55. Bastiaenen R, Pantazis A, Gonna H, et al. The ventricular ectopic QRS interval (VEQSI): Diagnosis of arrhythmogenic right ventricular cardiomyopathy in patients with incomplete disease expression. Heart Rhythm 2016;13: 1504-12.

56. Hoffmayer KS, Machado ON, Marcus GM, et al. Electrocardiographic comparison of ventricular arrhythmias in patients with arrhythmogenic right ventricular cardiomyopathy and right ventricular outflow tract tachycardia. J Am Coll Cardiol 2011;58:831-8.

57. Ainsworth CD, Skanes AC, Klein GJ, et al. Differentiating arrhythmogenic right ventricular cardiomyopathy from right ventricular outflow tract ventricular tachycardia using multilead QRS duration and axis. Heart Rhythm 2006; 3:416-23.

58. Novak J, Zorzi A, Castelletti S, et al. Electrocardiographic differentiation of idiopathic right ventricular outflow tract ectopy from early arrhythmogenic right ventricular cardiomyopathy. Europace 2017; 19:622-8.

59. Quarta G, Husain SI, Flett AS, et al. Arrhythmogenic right ventricular cardiomyopathy mimics: role of cardiovascular magnetic resonance. J Cardiovasc Magn Reson 2013;15:16.

60. Steckman DA, Schneider PM, Schuller JL, et al. Utility of cardiac magnetic resonance imaging to differentiate cardiac sarcoidosis from arrhythmogenic right ventricular cardiomyopathy. Am J Cardiol 2012;110:575-9.

61. Casella M, Pizzamiglio F, Dello Russo A, et al. Feasibility of combined unipolar and bipolar voltage maps to improve sensitivity of endomyocardial biopsy. Circ Arrhythm Electrophysiol 2015;8: 625-32.

62. Choudhary N, Tompkins C, Polonsky $\mathrm{B}$, et al. Clinical presentation and outcomes by sex in arrhythmogenic right ventricular cardiomyopathy: findings from the North American ARVC Registry. J Cardiovasc Electrophysiol 2016;27:555-62.
63. Bhonsale A, James CA, Tichnell C, et al. Incidence and predictors of implantable cardioverter-defibrillator therapy in patients with arrhythmogenic right ventricular dysplasia/cardiomyopathy undergoing implantable cardioverter-defibrillator implantation for primary prevention. J Am Coll Cardiol 2011;58:1485-96.

64. Corrado D, Calkins H, Link MS, et al. Prophylactic implantable defibrillator in patients with arrhythmogenic right ventricular cardiomyopathy/dysplasia and no prior ventricular fibrillation or sustained ventricular tachycardia. Circulation 2010;122:1144-52.

65. Brun F, Groeneweg JA, Gear K, et al. Risk Stratification in arrhythmic right ventricular cardiomyopathy without implantable cardioverter-defibrillators. JACC Clin Electrophysiol 2016;2:55864.

66. Pinamonti B, Dragos AM, Pyxaras SA, et al. Prognostic predictors in arrhythmogenic right ventricular cardiomyopathy: results from a 10-year registry. Eur Heart J 2011;32:1105-13.

67. Migliore F, Zorzi A, Silvano M, et al. Prognostic value of endocardial voltage mapping in patients with arrhythmogenic right ventricular cardiomyopathy/dysplasia. Circ Arrhythm Electrophysiol 2013;6:167-76.

68. Corrado D, Leoni L, Link MS, et al. Implantable cardioverter-defibrillator therapy for prevention of sudden death in patients with arrhythmogenic right ventricular cardiomyopathy/dysplasia. Circulation 2003;108:3084-91.

69. Schinkel AFL. Implantable cardioverter defibrillators in arrhythmogenic right ventricular dysplasia/cardiomyopathy: patient outcomes, incidence of appropriate and inappropriate interventions, and complications. Circ Arrhythm Electrophysiol 2013;6:562-8.

70. Burke MC, Gold MR, Knight BP, et al. Safety and efficacy of the totally subcutaneous implantable defibrillator: 2year results from a pooled analysis of the IDE Study and EFFORTLESS Registry. J Am Coll Cardiol 2015;65:1605-15.

71. Corrado D, Wichter T, Link MS, et al. Treatment of arrhythmogenic right ventricular cardiomyopathy/dysplasia. Circulation 2015;132:441-53.

72. Marcus GM, Glidden DV, Polonsky B, et al. Efficacy of antiarrhythmic drugs in arrhythmogenic right ventricular cardiomyopathy: a report from the North American ARVC Registry. J Am Coll Cardiol 2009;54:609-15.

73. Philips B, te Riele AS, Sawant A, et al. 
Outcomes and ventricular tachycardia recurrence characteristics after epicardial ablation of ventricular tachycardia in arrhythmogenic right ventricular dysplasia/cardiomyopathy. Heart Rhythm 2015;12:716-25.

74. Dalal D, Jain R, Tandri H, et al. Longterm efficacy of catheter ablation of ventricular tachycardia in patients with arrhythmogenic right ventricular dysplasia/cardiomyopathy. J Am Coll Cardiol 2007;50:432-40.

75. Santangeli P, Zado ES, Supple GE, et al. Long-term outcome with catheter ablation of ventricular tachycardia in patients with arrhythmogenic right ventricular cardiomyopathy. Circ Arrhythm Electrophysiol 2015;8:1413-21.

76. Tedford RJ, James C, Judge DP, et al. Cardiac transplantation in arrhythmogenic right ventricular dysplasia/cardiomyopathy. J Am Coll Cardiol 2012; 59:289-90.

77. Sawant AC, te Riele AS, Tichnell C et al. Safety of American Heart Association-recommended minimum exercise for desmosomal mutation carriers. Heart Rhythm 2016;13:199-207.

78. Priori SG, Blomström-Lundqvist C, Mazzanti A, et al. 2015 ESC Guidelines for the management of patients with ventricular arrhythmias and the prevention of sudden cardiac death. The Task Force for the Management of Patients with Ventricular Arrhythmias and the Prevention of Sudden Cardiac Death of the European Society of Cardiology. Eur Heart J 2015;36:2793-867.

79. Krahn AD, Healey JS, Gerull B, et al. The Canadian arrhythmogenic right ventricular cardiomyopathy registry: rationale, design, and preliminary recruitment. Can J Cardiol 2016;32: 1396-401. 